\title{
Investigation of Buffer Traps in AlGaN/GaN Heterostructure Field-Effect Transistors Using a Simple Test Structure
}

\author{
Seung Yup Jang ${ }^{1}$, Jong-Hoon Shin ${ }^{1}$, Eu Jin Hwang ${ }^{1}$, Hyo-Seung Choi ${ }^{2}$, \\ Hun Jeong', Sang-Hun Song, ${ }^{2, *}$, and Hyuck-In Kwon ${ }^{2, *}$
}

\begin{abstract}
We propose a new method which can extract the information about the electronic traps in the semi-insulating GaN buffer of AlGaN/GaN heterostructure field-effect transistors (HFETs) using a simple test structure. The proposed method has a merit in the easiness of fabricating the test structure. Moreover, the electric fields inside the test structure are very similar to those inside the actual transistor, so that we can extract the information of bulk traps which directly affect the current collapse behaviors of AlGaN/GaN HEFTs. By applying the proposed method to the GaN buffer structures with various unintentionally doped GaN channel thicknesses, we conclude that the incorporated carbon into the GaN back barrier layer is the dominant origin of the bulk trap which affects the current collapse behaviors of AlGaN/GaN HEFTs.
\end{abstract}

Index Terms-AIGaN/GaN HFET, semi-insulating GaN buffer, bulk trap, carbon-doped GaN back barrier

\section{INTRODUCTION}

Recently, $\mathrm{AlGaN} / \mathrm{GaN}$ heterostructure field-effect transistors (HFETs) fabricated on a silicon substrate are

Manuscript received Sep. 22, 2012; accepted Jan. 28, 2013 ${ }^{1}$ IGBT Part, System IC R\&D Laboratory, LG Electronics, 38, Baumoe-ro, Seocho-gu, Seoul 137-724, Korea

${ }^{2}$ School of Electrical and Electronics Engineering, Chung-Ang University, Seoul 156-756, Korea

E-mail : shsong@cau.ac.kr and hyuckin@cau.ac.kr attracting considerable attention as promising candidates for next generation microwave and power electronic devices [1]. However, there still remain some issues, including dynamic on-resistance degradation and current collapse which seriously limits the switching performance of devices [2]. In previous works, the formation of a front-side virtual gate associated with surface traps has been considered as the dominant mechanism causing the current collapse in $\mathrm{AlGaN} / \mathrm{GaN}$ HFETs [2]. However, recent reports show that the bulk traps in the semi-insulating GaN buffer also can cause the serious current collapse in AlGaN/GaN HFETs [3]. Previously, the bulk traps in the semi-insulating GaN buffer have been mainly investigated using the capacitance-mode deep level transient spectroscopy (CDLTS) [4], the backgating current transient spectroscopy $[5,6]$, and the drain current transient method [7-9]. CDLTS is a powerful and well-established technique for the extraction of bulk traps in semiconductor devices. However, since it generally requires large area Schottky diodes, C-DLTS is difficult to apply to actual transistors due to their small gate capacitance. Considering that the distribution of the electric field is quite different in diodes and in actual transistors, the obtained results from C-DLTS cannot give clear information about the location of electronic traps in HFET structures. The backgating current transient spectroscopy is the method which has been used to investigate the bulk trap in the metalsemiconductor field effect transistors and HFETs. For HFETs, the backgating depletion region and therefore the influence of the substrate voltage is limited below the 
two dimensional electron gas (2DEG), therefore, all related effects have their origin in the bulk without contribution of surface effects. This method has a merit that it does not require a large area device. However, electronic trap extracted from this method can be different from the one which affects the current collapse behavior of the device, because the electric field distribution within the device during the application of the substrate bias is different from that during the operation of the transistor. As a more effective method to extract the bulk trap in AlGaN/GaN HFETs, the drain current transient method was suggested by Joh et al. [7]. They expose the AlGaN/GaN HFETs to the on-state stress (high $\mathrm{I}_{\mathrm{D}}$ and relatively high $\mathrm{V}_{\mathrm{DS}}, \mathrm{V}_{\mathrm{GS}}=0 \mathrm{~V}$ and $\mathrm{V}_{\mathrm{DS}}=10 \mathrm{~V}$ in [7]) for a while, and observe the subsequent drain current transient in the linear regime $\left(\mathrm{V}_{\mathrm{GS}}=1 \mathrm{~V}\right.$ and $\mathrm{V}_{\mathrm{DS}}=0.5 \mathrm{~V}$ in [7]). In this method, the information about the trap can be obtained from the recovery characteristics of $I_{D}$ due to the detrapping of electrons which were captured by the traps during the onstate stress. Although this method can extract the information about the electronic traps directly from the actual transistors, it has a drawback of discerning the location of the extracted traps between the surface and the bulk, because the electrons are trapped not only by the bulk trap but by the surface trap during the on-state stress due to the relatively high $\mathrm{V}_{\mathrm{DG}}$. In this letter, we propose a new method which can selectively extract the information about the electronic traps in the semiinsulating GaN buffer of AlGaN/GaN HFETs using a simple test structure, and investigate the bulk traps in the GaN buffer structures with various unintentionally doped (UID) GaN channel thicknesses based on the proposed method.

\section{TEST STRUCTURE FABRicATION \& MEASUREMENT SCHEME}

Fig. 1(a) shows the cross sectional view of the proposed test structure to investigate the electronic traps in the semi-insulating GaN buffer of AlGaN/GaN HFETs. The samples were grown by metal-organic chemical vapor deposition (MOCVD) on 6-in Si substrates, and carbon doping of GaN buffer is achieved without an additional carbon source. UID GaN channel layers with various thicknesses $(200-1000 \mathrm{~nm})$ were followed by a (a)

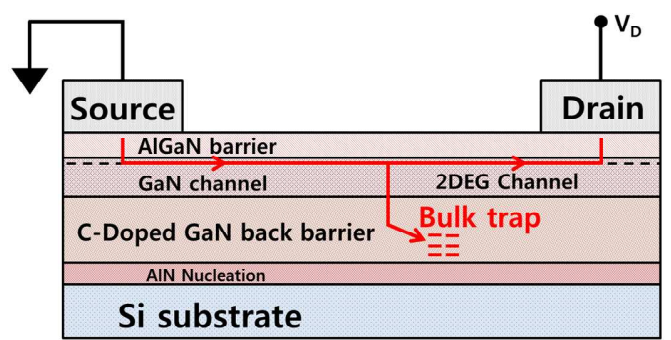

(b)

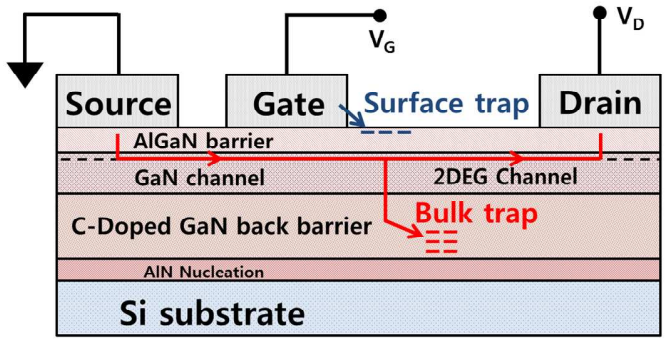

Fig. 1. Cross sectional view of the (a) proposed test structure, (b) $\mathrm{AlGaN} / \mathrm{GaN}$ HFET. Arrows indicate the electron flow. Because there is no gate electrode in the proposed test structure, the electrons are not captured by the surface traps in the gatedrain access region during the on-state stress, and the recovery characteristics of $I_{D}$ can be attributed solely to the detrapping of electrons from the traps located in the GaN buffer.

GaN:C buffer structure $\left(2 \mu \mathrm{m},[\mathrm{C}] \sim 10^{19} \mathrm{~cm}^{-3}\right)$. A 25 nm-thick $\mathrm{Al}_{0.25} \mathrm{Ga}_{0.75} \mathrm{~N}$ layer was then grown on a UID GaN channel layer to form the $2 \mathrm{DEG}$, and $\mathrm{Ti} / \mathrm{Al} / \mathrm{Pd} / \mathrm{Au}$ metal stacks were deposited and annealed at $800{ }^{\circ} \mathrm{C}$ in $\mathrm{N}_{2}$ atmosphere for source and drain electrodes. The width (W) and length (L) of the test structure were designed to be $200 \mu \mathrm{m}$ and $30 \mu \mathrm{m}$, respectively.

In the proposed method, the information about electronic trap in the GaN buffer can be extracted by monitoring the time transient of $I_{D}$ right after reducing the magnitude of $\mathrm{V}_{\mathrm{DS}}$. When $\mathrm{V}_{\mathrm{DS}}$ is high, relatively large $I_{D}$ flows between source and drain electrodes through the channel and bulk of the test structure because the AlGaN/GaN HFET exhibits the normally-on characteristics, and electrons are captured by the bulk trap during this operation. After lowering $\mathrm{V}_{\mathrm{DS}}$, the trapped electrons in the GaN buffer are detrapping from the bulk trap, and $\mathrm{I}_{\mathrm{D}}$ continuously increases with a time.

By observing these recovery characteristics of $I_{D}$ at various temperatures, we can extract the information of GaN bulk trap. As commented in the introduction part, electrons are captured by the traps located not only in the GaN buffer but in the surface when the $\mathrm{AlGaN} / \mathrm{GaN}$ HEFTs are exposed to the on-state stress in the 
conventional drain current transient method (Fig. 1(b)). However, because there is no gate electrode in the proposed test structure, the electrons are not captured by the surface traps in the gate-drain access region during the on-state stress (Fig. 1(a)), and we can attribute the recovery characteristics of $I_{D}$ solely to the detrapping of electrons from the traps located in the GaN buffer. The suggested method has a merit that it is very simple to fabricate the test structure and easy to apply. Moreover, the electric fields inside the test structure are very similar to those inside the actual transistor, so we can extract the information of bulk traps which directly affect the electrical behaviors of AlGaN/GaN HEFTs.

\section{RESULTS AND DisCUSSION}

Fig. 2(a) depicts the time transients of $I_{D}$ measured at $\mathrm{V}_{\mathrm{DS}}=0.5 \mathrm{~V}$ right after exposing the test structure (UID $\mathrm{GaN}$ channel thickness : $200 \mathrm{~nm}$ ) to the on-state stress $\left(\mathrm{V}_{\mathrm{DS}}=10 \mathrm{~V}\right)$ for $10 \mathrm{~s}$. Measurements were made at various temperatures ranging from $\mathrm{RT}$ to $80{ }^{\circ} \mathrm{C}$ using the Agilent $4156 \mathrm{C}$ semiconductor parameter analyzer. The test structure was illuminated by microscope light for 30 $\mathrm{s}$ before each measurement to recover the initial condition of the device. Fig. 2(a) shows that $I_{D}$ increases with a time during the measurement at RT, and it is accelerated as the temperature increases. This phenomenon can be attributed to the accelerated detrapping process of captured electrons at higher temperatures. Fig. 2(b) shows the Arrhenius plot depicted using the extracted time constants from the results in Fig. 2(a). The multiple-exponential fitting method was used to extract the time constants from $\mathrm{I}_{\mathrm{D}}$ transients at each temperature [7], and the activation energy $\left(\mathrm{E}_{\mathrm{a}}\right)$ is determined to be $\sim 0.25 \mathrm{eV}$ from the Arrhenius plot in Fig. 2(b). Previously in theoretical studies, the formation of donor states with an energy level of $\sim 0.2 \mathrm{eV}$ below the conduction band edge was expected when $\mathrm{C}$ substitutes $\mathrm{Ga}\left(\mathrm{C}_{\mathrm{Ga}}\right)$ in carbon doped $\mathrm{GaN}(\mathrm{GaN}: \mathrm{C})$ [10, 11]. The calculated $E_{a}$ from Fig. 2(b) is consistent with this result, and it implies that the incorporated carbon into $\mathrm{GaN}$ to prevent punchthrough currents at high electric field is the possible origin of the bulk trap which affecting the electrical behaviors of AlGaN/GaN HEFTs even though the carbon-doped $\mathrm{GaN}$ back barrier is located 200-nm below 2DEG channel. Fig. 2(a) also
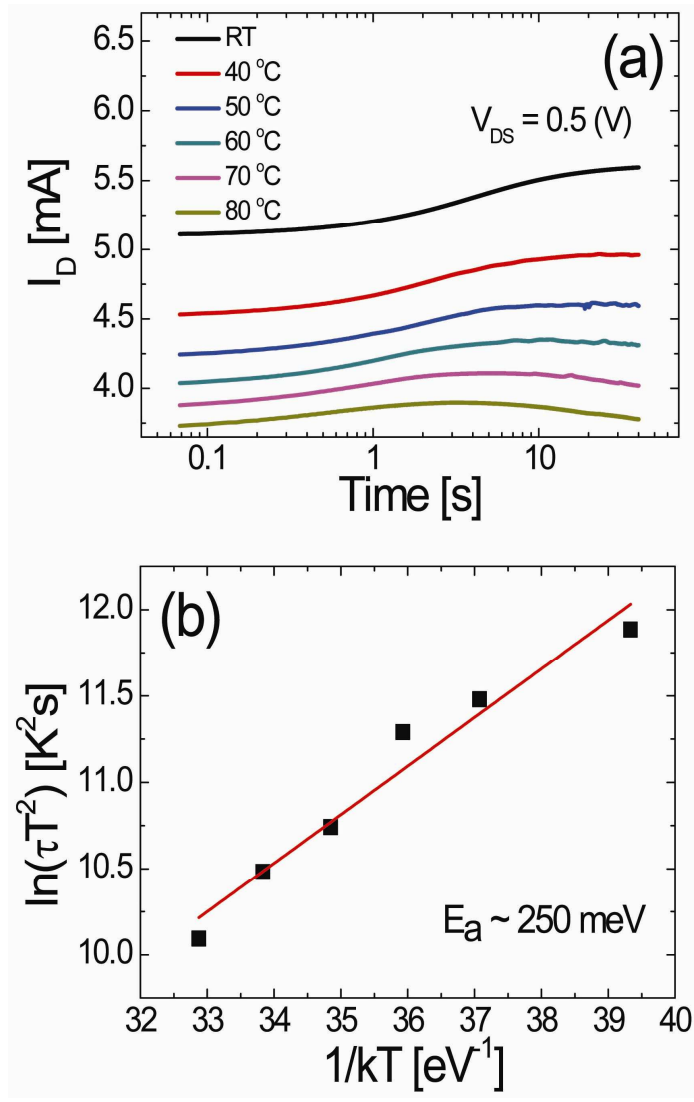

Fig. 2. (a) Time transients of $I_{D}$ measured at $V_{D S}=0.5 \mathrm{~V}$ right after exposing the test structure (UID GaN channel thickness: $200 \mathrm{~nm})$ to the on-state stress $\left(\mathrm{V}_{\mathrm{DS}}=10 \mathrm{~V}\right)$ for $10 \mathrm{~s}$ at various temperatures ranging from RT to $80{ }^{\circ} \mathrm{C}$, (b) Arrhenius plot depicted using the extracted time constants from the results in Fig. 2(a). Time constants are extracted as $3.82,2.35,2.16,1.16$, 0.87 , and $0.66 \mathrm{~s}$ at RT, $40,50,60,70$, and $80{ }^{\circ} \mathrm{C}$, respectively.

shows the slight decrease of $I_{D}$ with a time at high temperatures, it may be attributed to the re-trapping of electrons during the current transient measurements.

Fig. 3 depicts the time transients of normalized $I_{D}$ measured for test structures with different UID GaN channel thicknesses at RT. Measurements were made at a same condition with that in Fig. 2(a). Fig. 3 shows that the magnitude of detrapping current is much reduced when UID GaN channel thickness increases. Moreover, the detrapping current becomes negligible in test structures without a carbon-doped GaN back barrier. This result confirms the conclusion of Fig. 2 that the carbon-doped GaN back barrier is the dominant source of bulk trap in fabricated AlGaN/GaN test structures, and shows that the current collapse behavior caused from the carbon doping cannot be perfectly eliminated even when the vertical spacing between the 2DEG channel and the 


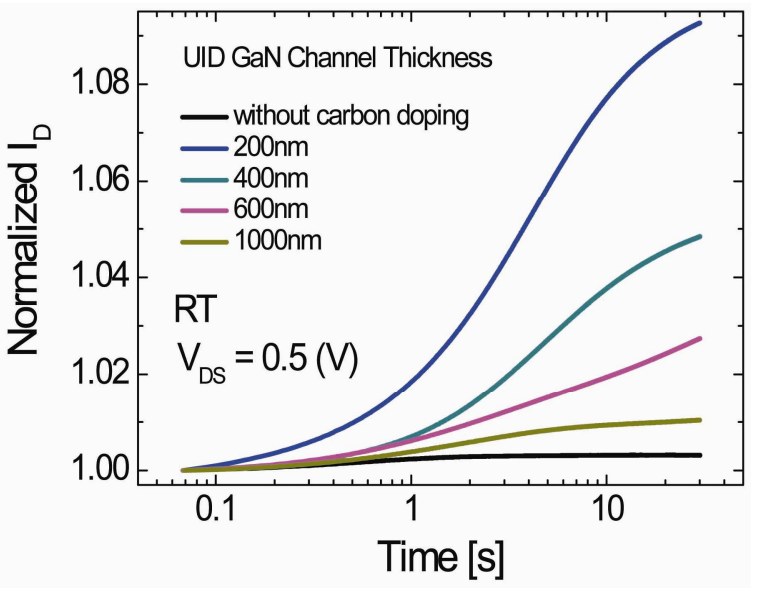

Fig. 3. Time transients of normalized $I_{D}$ measured for test structures with different UID GaN channel thicknesses at RT. Measurements were made at a same condition with that in Fig. 2(a).

carbon-doped GaN back barrier is $1 \mu \mathrm{m}$.

Fig. 4 compares the time transients of normalized $I_{D}$ measured for AlGaN/GaN HFETs (Fig. 4(a)) and test structures (Fig. 4(b)) with a UID GaN channel thickness of 200 and $400 \mathrm{~nm}$ at RT. The AlGaN/GaN HFETs are fabricated by forming the $\mathrm{p}-\mathrm{GaN}$ gate on the AlGaN/GaN heterostructure. The HFET and test structure with a same UID GaN channel thickness were fabricated on the same wafer and source-to-drain distance of the HFET was designed to be same with that of the test structure. Fig. 4(c) depicts the transfer curves measured from the fabricated AlGaN/GaN HFETs. In AlGaN/GaN HFETs, the time transient measurements were made after changing the bias point $\left(\mathrm{V}_{\mathrm{GS}}, \mathrm{V}_{\mathrm{DS}}\right)$ from $(2 \mathrm{~V}, 10 \mathrm{~V})$ to $(2 \mathrm{~V}, 0.5 \mathrm{~V})$. From the results of Fig. 4(a) and (b), we can observe that the detrapping transient of normalized $\mathrm{I}_{\mathrm{D}}$ exhibit a similar shape in the HFET and test structure when a UID GaN channel thickness is same, which implies that the relatively slow current transient phenomenon (time constant $(\tau)>0.1 \mathrm{~s}$ ) in fabricated $\mathrm{AlGaN} / \mathrm{GaN}$ HFET is mainly due to the electron detrapping from the bulk trap located in the carbondoped $\mathrm{GaN}$ back barrier.

\section{CONCLUSIONS}

In this letter, we propose the new method which can investigate the buffer traps in the AlGaN/GaN HFETs using a simple test structure. The operation principle of
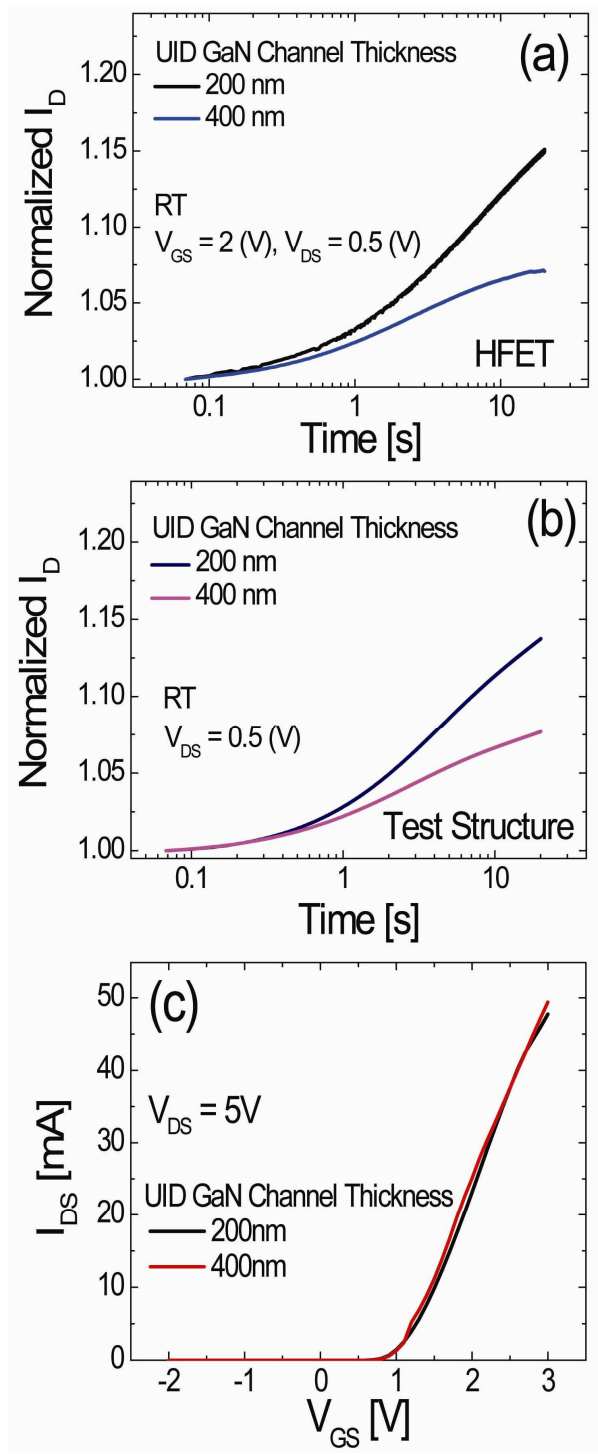

Fig. 4. Time transients of normalized $I_{D}$ measured for (a) AlGaN/GaN HFETs, (b) test structures with a UID GaN channel thickness of 200 and $400 \mathrm{~nm}$ at RT. (c) Transfer curves measured from the fabricated AlGaN/GaN HFETs with a UID $\mathrm{GaN}$ channel thickness of 200 and $400 \mathrm{~nm}$.

the proposed method is similar with that of the conventional drain current transient method, but we can selectively extract the information about bulk trap using the proposed method. The electric fields inside the test structure are very similar to those inside the actual HFET, so we can extract the information of bulk traps which directly affect the current collapse behaviors of AlGaN/GaN HEFTs. From the application of the proposed method to GaN buffer structures with various UID GaN channel thicknesses, carbon-doped GaN back barrier is concluded as the dominant source of the bulk 
trap in fabricated AlGaN/GaN test structures even when the vertical spacing between the 2DEG channel and the carbon-doped GaN back barrier is $1 \mu \mathrm{m}$.

\section{ACKNOWLEDGMENTS}

This work was supported by the Chung-Ang University Research Grants in 2013.

\section{REFERENCES}

[1] M. Ishida, T. Ueda, T. Tanaka, and D. Ueda, "GaN on Si technologies for power switching devices," IEEE Trans. Electron Devices, vol. 60, no. 10, pp. 3053-3059, Oct. 2013.

[2] S. Arulkumaran, G. I. Ng, and Z. H. Liu, "Effect of gate-source and gate-drain Si3N4 passivation on current collapse in $\mathrm{AlGaN} / \mathrm{GaN}$ high-electronmobility transistors on silicon," Appl. Phys. Lett., vol. 90, no. 17, pp. 173504-1-173504-3, Apr. 2007.

[3] M. J. Uren, J. Möreke, and M. Kuball, "Buffer design to minimize current collapse in GaN/AlGaN HFETs," IEEE Trans. Electron Devices, vol. 59, no. 12, pp. 3327-3333, Dec. 2012.

[4] Z. Q. Fang, B. Claflin, D. C. Look, D. S. Green, R. Vetury, "Deep traps in AlGaN/GaN heterostructures studied by deep level transient spectroscopy: Effect of carbon concentration in GaN buffer layers," J. Appl. Phys., vol. 108, no. 6, pp. 063706-1-0637066, Sep. 2010.

[5] M. Marso, M. Wolter, P. Javorka, P. kordos, and H. Luth, "Investigation of buffer traps in an $\mathrm{AlGaN} / \mathrm{GaN} / \mathrm{Si}$ high electron mobility transistor by backgating current deep level transient spectroscopy," Appl. Phys. Lett., vol. 82, no. 4, pp. 633-635, Jan. 2003.

[6] M. J. Uren, M. Silverstri, M. Casar, G. A. M. Hurkx, J. A. Croon, J. Sonsky, and M. Kuball, "Intentionally carbon-doped AlGaN/GaN HEMTs: Necessaty for vertical leakage paths," IEEE Electron Device Lett., vol. 35, no. 3, pp. 327-329, Mar. 2014.

[7] J. Joh and J. del Alamo, "Impact of electrical degradation on trapping characteristics of GaN high electron mobility transistors," in IEDM Tech. Dig., 2008, pp. 461-464.
[8] J. Joh and J. del Alamo, "A current-transient methodology for trap analysis for $\mathrm{GaN}$ high electron mobility transistors," IEEE Trans. Electron Devices, vol. 58, no. 1, pp. 132-140, Jan. 2011.

[9] D. Bisi, M. Meneghini, C. de Santi, A. Chini, M. Dammann, P. Bruckner, M. Mikulla, G. Meneghesso, and E. Zanoni, "Deep-level characterization in GaN HEMTs-Part I: Advantages and limitations of drain current transient measurements," IEEE Trans. Electron Devices, vol. 60, no. 10, pp. 3166-3175, Oct. 2013.

[10] P. Boguslawski and J. Bernholc, "Doping properties of $\mathrm{C}, \mathrm{Si}$, Ge impurities in $\mathrm{GaN}$ and AlN," Phys. Rev. B, vol. 56, no. 15, pp. 9496-9505, Jul. 1997.

[11] A. F. Wright, "Substitutional and interstitial carbon in wurtzite GaN," J. Appl. Phys., vol. 92, no. 5, pp. 2575-2585, Sep. 2002.

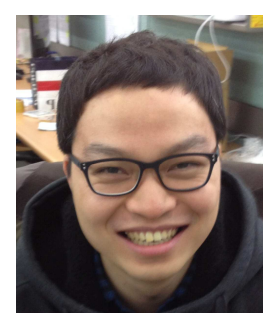

Seung Yup Jang received B.S and Ph.D degree in physics from Seoul National University, Seoul, Korea in 2004 and 2010, respectively. He is currently senior research engineer in LG electronics. His research interest is evaluation and analysis of $\mathrm{GaN}$

power device.

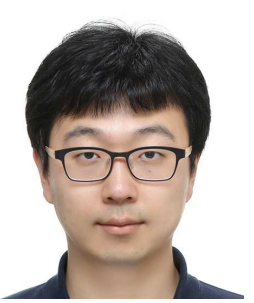

Jong-Hoon Shin was born in Seoul, Korea, in 1983. He received the B.S and M.S degree in physics from Seoul National University, Seoul, Korea in 2008 and 2010 respectively. He is currently doing research in LG Electronics, where his research interest is device physics and switching characteristics of $\mathrm{GaN}$ power devices.

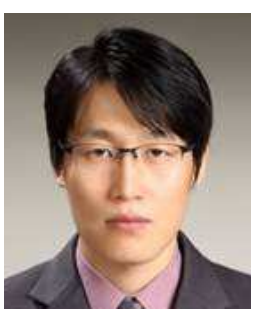

Eu Jin Hwang received a M.S. degree in semiconductor physics from Chonbuk National University, in 2003. He is currently chief research engineer in $\mathrm{LG}$ electronics. His research interest is growth of compound semiconductor for power semiconductor device. 


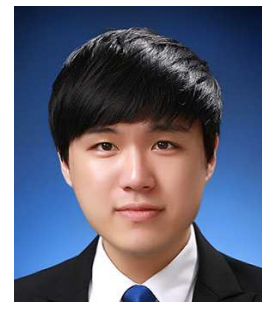

Hyo-Seung Choi received a bachelor's degree in Electrical and Electronics Engineering from ChungAng University, in 2013. He is currently a graduate school student in the School of Electrical and Electronics Engineering at Chung-Ang University in Seoul. His current research interests include GaN-based power semiconductor devices.

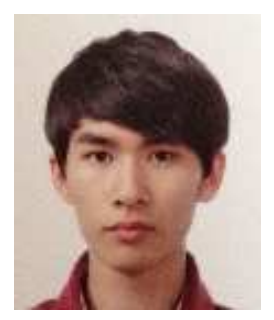

Hun Jeong received a bachelor's degree in Electrical and Electronics Engineering from Chung-Ang University, in 2014. He is currently a graduate school student in the School of Electrical and Electronics Engineering at Chung-Ang University in Seoul. His current research interests include GaNbased power semiconductor devices.

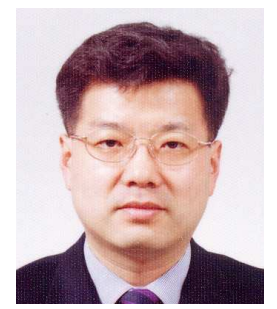

Sang-Hun Song received his BS degree in Electronics Engineering from Seoul National University in 1986 and his MA and Ph.D. degrees both from Princeton University in 1988 and 1997 respectively. His doctoral research studied on magneto optical and magneto transport properties of the 2 dimentional carriers in strained semiconductor layers. In 1997, he joined LG Semincon Co., Ltd. as a DRAM circuit designer. In 2001, he joined the School of Electrical and Electronics Engineering at Chung-Ang University in Seoul, where he is now a professor. In 2007, he was a visiting scholar to GLAM at Stanford University. His research interests include semiconductor physics and devices, memory devices, nanoelectronics and novel techniques in semiconductor characterization. Recently, he is working on sensors that can detect force and mass.

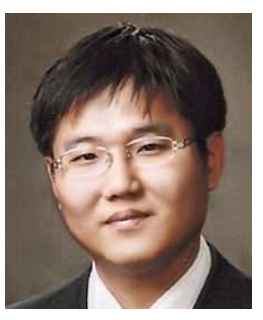

Hyuck-In Kwon received the B.S., M.S., and Ph.D. degrees in electrical engineering from Seoul National University, Seoul, in 1999, 2001, and 2005, respectively. From August 2004 to March 2006, he was a Research Associate with the University of Illinois, Urbana. In 2006, he joined the System LSI Division, Samsung Electronics Company, Korea, where he was a Senior Engineer with the Image Development Team. From September 2007 to February 2010, he worked for the School of Electronic Engineering in Daegu University as a Full-Time Lecturer and an assistant professor. Since 2010, he has been with Chung-Ang University, Seoul, Korea, where he is currently an associate professor in the School of Electrical and Electronics Engineering. His research interests include CMOS active pixel image sensors, oxide thin-film transistors, GaN-based power devices, and silicon nanotechnologies. 